Research Article

\title{
Combat Network Synchronization of UCAV Formation Based on RTBA Model
}

\author{
Chengwei Ruan, Haiyan Yang, Lei Yu, and Yingxin Kou \\ Aeronautics and Astronautics Engineering College, Air Force Engineering University, Xian 710038, China \\ Correspondence should be addressed to Haiyan Yang; yanghy07@yeah.net
}

Received 16 July 2016; Accepted 5 September 2016

Academic Editor: Zhao Cheng

Copyright (C) 2016 Chengwei Ruan et al. This is an open access article distributed under the Creative Commons Attribution License, which permits unrestricted use, distribution, and reproduction in any medium, provided the original work is properly cited.

The paper aims at developing an efficient method to acquire a proper UCAV formation structure with robust and synchronized features. Here we introduce the RTBA (Route Temporary Blindness Avoidance) model to keep the structure stable and the HPSO (hybrid particle swarm optimization) method is given to find an optimal synchronized formation. The major contributions include the following: (1) setting up the dynamic hierarchy topologic structure of UCAV formation; (2) the RTB phenomenon is described and the RTBA model is put forward; (3) the node choosing rules are used to keep the invulnerability of the formation and the detective information quantifying method is given to measure the effectiveness of the connected nodes; and (4) the hybrid particle swarm optimization method is given to find an optimal synchronized topologic structure. According to the related principles and models, the simulations are given in the end, and the results show that the simplification of the model is available in engineering, and the RTBA model is useful to solve the real problems in combat in some degree.

\section{Introduction}

Since the control theory and intelligent technology have acquired significant advances, research on multiple unmanned combat aerial vehicles (UCAVs) has attracted more and more attentions in recent years. The cooperative problems [1], control command problems [2-4], mission assignment problems [5-7], and route planning problems $[8,9]$ have become the focus among researchers. The structure of UCAV formation has also been focused on in recent years. The frequent exchanging information among different UCAVs during the mission process needs a robust and efficient structure to keep the systems up. A robust status needs an adaptive structure both in physical level and in functional level and an efficient job among UCAVs needs a consistent recognition towards the same combat situation.

Many researches concerned about the UCAV formation have assumed that all the UCAV units had their information completely shared, and the communication interruptions from both the enemies and the measurement errors are neglected, such as $[10,11]$. But, in real combat, the UCAV units can only get limited information about the situation due to the limitations of current communicating abilities. Just a few references have described the UCAV model with a local knowledge mastered by the combat units. Reference [12] has designed a new distributed auction algorithm based on the neighbor knowledge. Reference [13] puts forward a cooperative missile guiding rule based on the local communication and the features of heterogeneous structure. Reference [14] gives a new distributed auction algorithm with a limited communication ability. Though these researches have brought us many valuable results which may be useful to solve some problems in real society, the researches on topology structure and the dynamic model still need to be analyzed.

Route Temporary Blindness (RTB) problem [15] which may lead to communication interruption may also be very important to have an effect on the robustness of the UCAV formation. The communication among UCAVs during the continuous mission executing process may lead to RTB problem, and the RTB problem may force the formation to upgrade its structure. In order to get an optimal UCAV formation, we put forward a method in this study to solve the RTB problem and get an optimal structure with efficient synchronized features.

The paper is organized as follows: Section 1 is introduction. Section 2 presents the dynamic topology model of 


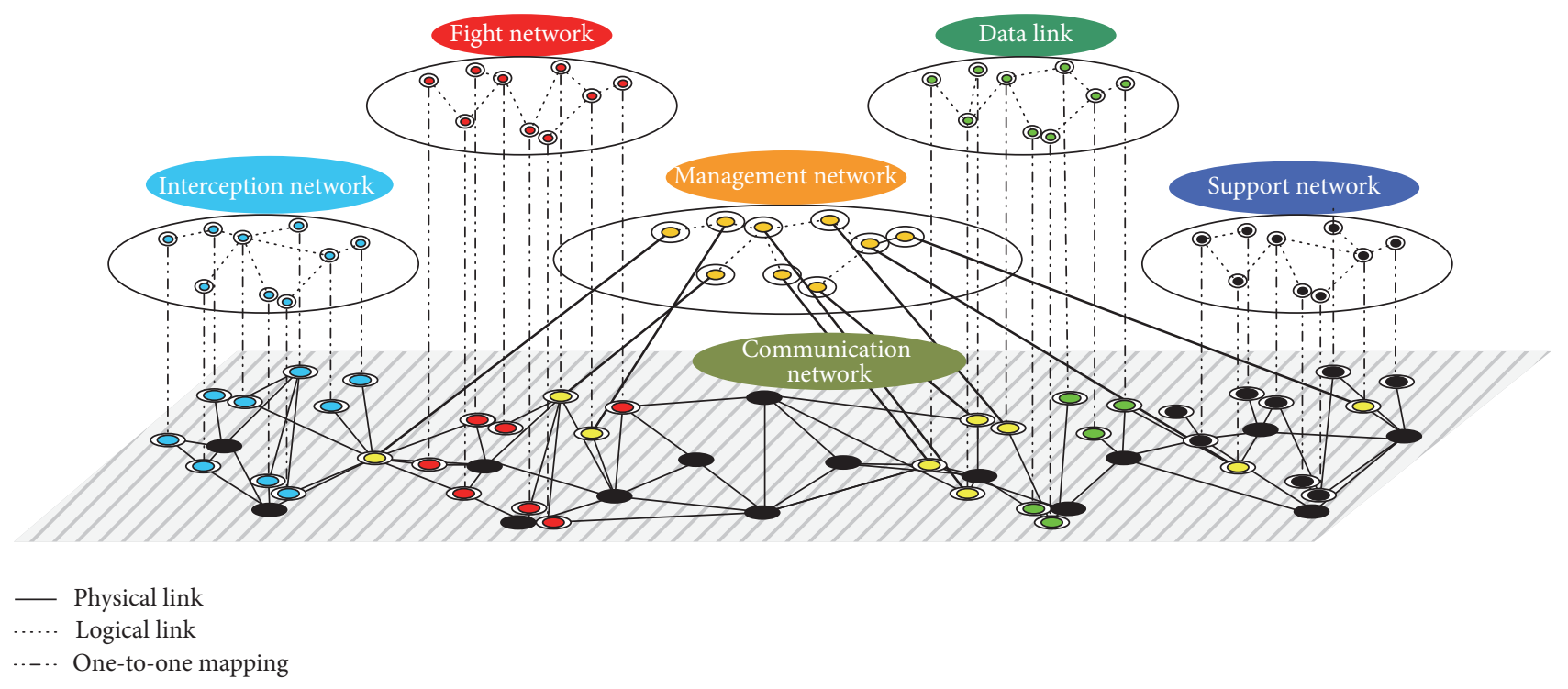

FIGURE 1: Relationships between physical and functional nodes in UCAV formation.

UCAV formation and its node survival model. Section 3 describes the rout blindness problem and its solution. Section 4 puts forward an optimal algorithm aiming at finding a better structure with a more stable and synchronized features. Section 5 gives the simulation results and Section 6 is conclusion.

\section{Dynamic Topology Modeling of UCAV Net Constructing}

In order to solve the cooperative problems in UCAV cooperation, we need to better understand the structure of the UCAV formation both in physical and in functional levels. The structure of UCAV formation can be described as $\mathbf{G}=$ $(\mathbf{P}, \mathbf{F}), \mathbf{P}$ is the variable set of physical structure which can be described as $\mathbf{P}=(\mathbf{V}, \mathbf{E}, \mathbf{C}), \mathbf{V}$ is the set of UCAV nodes, and $\mathrm{E}$ means the connections between different nodes. Each edge $e_{i} \in \mathbf{E}$ can find two relative nodes $\left(v_{j}, v_{k}\right)$ in $\mathbf{V}$. C is the feature set of UCAV formation, $C_{n}(k) \in \mathbf{C}\left(n=1,2, \ldots, n_{\max } ; k=\right.$ $\left.1,2, \ldots, k_{\max }\right), n_{\max }$ is the maximum number of UCAVs, and $k_{\max }$ is the maximum number of the kinds of UCAV features. $\mathbf{F}$ is the variable set of functional structures which is constructed as $\mathbf{F}=(\mathbf{M}, \mathbf{R}), \mathbf{M}$ is the number of kinds of different missions, and $\mathbf{R}$ represents a set of logic relationships between different functional nodes.

2.1. Physical and Functional Networks. The fast developed technologies have greatly expanded the functions of UCAVs, and different cooperative relationships may bring various functional UCAV formations to satisfy the continuously changing missions. In order to efficiently complete the given missions, we need to analyze the relationships of the net constructing structures between physical and functional levels in UCAV formations.

From Figure 1, we can see that one SoS (system of systems) of UCAV can be divided into several parts according to the needs of missions, such as fight network, interception network, support network, management network, and communication network. The latter one is communication network which can be regarded as the physical network and other networks are functional networks which are in close relationships with the physical network. For most real UCAV systems, their functional connections and physical connections are not equivalent.

2.1.1. Heterogeneity of UCAVs. For UCAV formation, each single UCAV has its own special features which match different kinds of functional networks, we suppose that there are $N$ kinds of UCAVs, and the number of UCAVs $k$ is $\left|R_{k}\right|$, the state space is $S_{k}$, and the relative action state is $A_{k}$. Then the mapping formula can be described as

$$
\prod_{k=1}^{N} S_{k}{ }^{\left|R_{k}\right|} \longrightarrow \prod_{k=1}^{N} A_{k}{ }^{\left|R_{k}\right|} \text {. }
$$

The transforming description can be seen in Figure 2 .

In Figure 2, $\mathbf{E}$ means the environment, and the other variables can be found in Section 2. Figure 2 is a typical OODA ring which can also reflect the transforming process of constructing the physical net to complete the task of functional net. A stable and static physical net can be useful to keep the high efficiency of UCAV formation. But, in real combat, the dynamic environment and other continuous changing factors may need a dynamic structure to support UCAV formation.

2.1.2. Time-Varying Features of UCAV Structure. According to the analysis in Section 1, it is easy to understand the importance of a dynamic structure of UCAV formation. The description of the dynamic model can be seen in Figure 3.

Figure 3 shows the dynamic process of the transformation in the net constructing structure. During different time areas, 


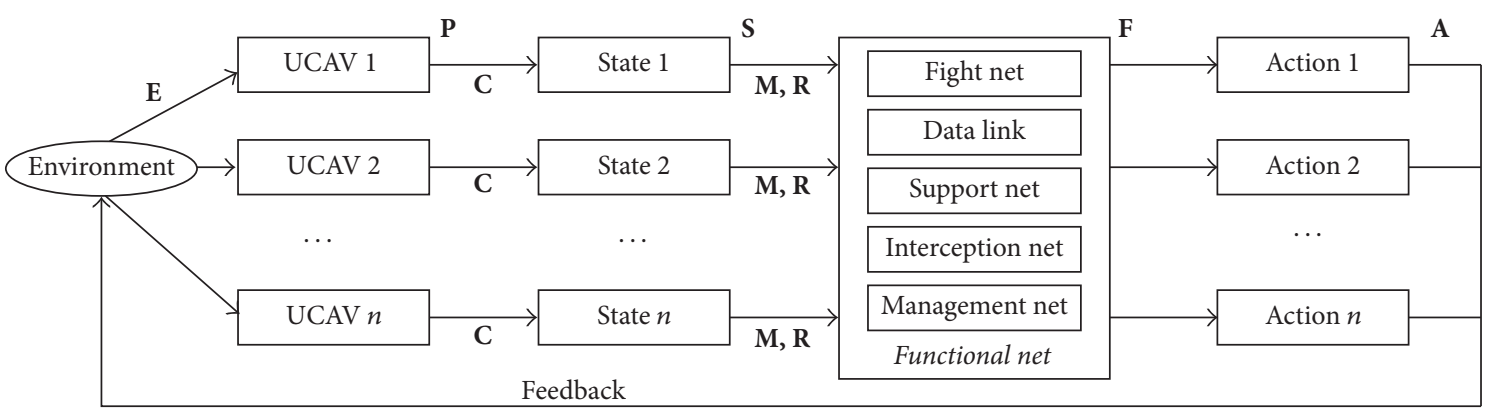

FIGURE 2: State transformation description of UCAV formation.

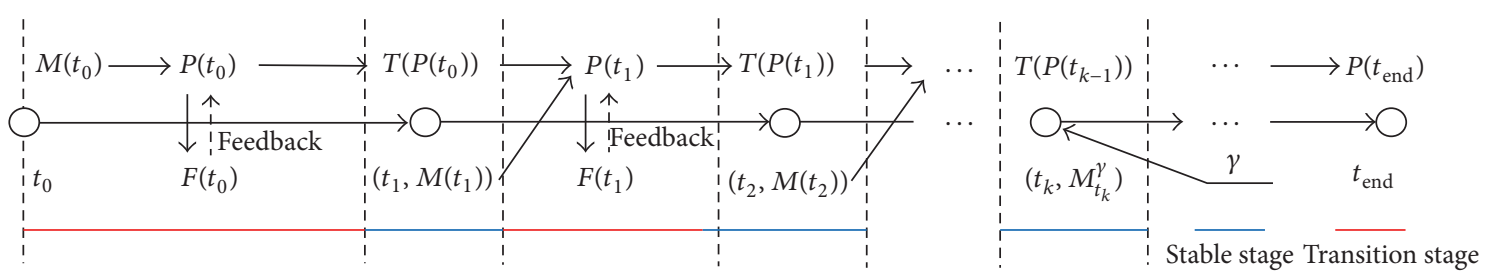

FIGURE 3: Dynamic process description of formation.

the physical structure is different due to the needs of the missions and the features of each UCAV. $P(t)$ is the timevarying function of physical structure which is driven by the missions. Different missions may stimulate different physical structure which can affect the style of functional structure $F(t)$ greatly. The results coming from functional structure may also reaffect the physical construction. The physical structure will change at time $t_{n}(n=1,2, \ldots, N)$, and $\gamma$ in Figure 3 means the unexpected missions which may cause the changes of the physical structure. In particular, time between $t_{k}$ and $t_{k+1}$ is not a constant, and it depends on the needs of the relative missions.

Formula (2) can describe the dynamic process:

$$
\begin{aligned}
P(t) & =P(t)+\Delta P, \quad t \in\left(t_{k}, t_{k+1}\right), \quad k=1,2, \ldots, N \\
P\left(t_{k+1}\right) & =T\left(P\left(t_{k}\right)\right), \quad k=1,2, \ldots, N \\
P\left(t_{0}\right) & =\left(V_{0}, E_{0}, C_{0}\right) .
\end{aligned}
$$

From formula (2), we can see that, during the stable stage, the physical structure keeps a stable status without considering the error $\Delta P$, which can be described as $\Delta P=$ $f(\Delta V, \Delta X, \Delta Y, \Delta Z), \Delta V, \Delta X, \Delta Y$, and $\Delta Z$ are the velocity and position errors of each UCAV. At time $t_{k}, k=1,2, \ldots, N$, the physical structure may change due to the new coming missions. $T$ is the transforming function which can help the system to complete the update. $P\left(t_{0}\right)$ is the initial description of the structure which is decided by the given mission.

\subsection{Node Survival Model of Multi-UCAV Formation. Accord-} ing to [16-18], we can conclude some obvious features of complex system. They are as follows:

(i) The component systems achieve well-substantiated purposes independently even if detached from the overall system. (ii) The whole function of a complex system cannot be easily composed of every single subsystem $(1+1>2)$.

(iii) It has some intelligent features in some degree which can complete a special mission by predicting the situation using the current information.

From the features we mentioned above, we can regard the UCAV formation as a complex system. Then the vulnerability feature should be analyzed in order to keep the system stable.

The failure probability function can be described as [19]

$$
W_{\alpha}\left(k_{i}\right)=\frac{k_{i}^{\alpha}}{\sum_{i=1}^{N} k_{i}^{\alpha}}, \quad(-\infty<\alpha<\infty),
$$

where $k_{i}$ denotes the degree of the node $i$ and $\alpha$ can be regarded as the measure of the knowledge on the network structure. There are four kinds of conditions:

(i) $\alpha<0$, which means that the attacker masters more information about the nodes with low degree. Node with low degree may be more vulnerable and the attacker may use the low-node-first attack strategy.

(ii) $\alpha>0$, which means that the attacker masters more information about the nodes with high degree. Node with high degree may be more vulnerable and the attacker may use the high-node-first attack strategy.

(iii) $\alpha=0$, which means the random attack.

(iv) $\alpha=\infty$, which means the deliberate attack.

\section{Description of Route Temporary Blindness Problem in UCAV}

In Figure 1, the importance of physical and functional structure of UCAV formation has been described. We can 


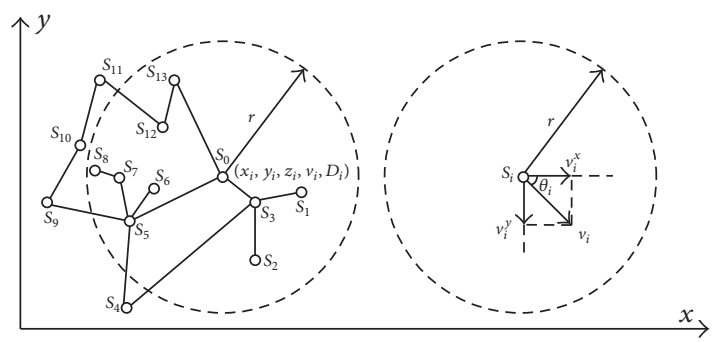

FIgURE 4: Description of RTB problem.

easily find that the physical network is the foundation of the whole mission. And a proper physical structure is the most important factor for the formation to complete the given mission. And, in this paper, we regard the communication network as the physical network. Thus, the Route Temporary Blindness problem comes.

3.1. Problem Statement. When cooperating with other UCAVs during a mission, a single UCAV needs the information from its neighbors. A stable and reliable neighbor may be of great importance to keep the stable status of the whole structure.

The Route Temporary Blindness problem may be caused in different stages:

(1) During relatively stable stage, the problem may be caused by $\triangle P$. The accumulated errors in position may disturb the normal prediction of the single UCAV, and the deviated position within an information updated cycle may lead to the overranging of the communication radius. Then the chosen neighbor node may be unavailable and the communication in next cycle may be broken.

(2) At the mission transforming moment, the problem may be caused by the change of the dynamic physical structure. The vulnerability of the nodes in different mission processes may get different values which may cause the changes in position and connection among UCAVs. When a mission changes, the highest degree neighbor may change from the most vulnerable node to the least vulnerable node. So the neighbor needs to be changed in order to protect the formation from the future temporary blindness problem.

From Figure $4, S_{i}$ means the UCAV node in formation, $S_{0}$ is the node which has been researched on, and $\left(x_{i}, y_{i}, z_{i}, v_{i}, D_{i}\right)$ is the information communicated between $S_{0}$ and its neighbors. $x_{i}, y_{i}$, and $z_{i}$ are the position information, $v_{i}$ is the velocity, and $D_{i}$ is the degree of its neighbors.

\subsection{RTBA Model}

3.2.1. Route Temporary Blindness Physical Avoidance (RTBPA) Model. In order to avoid the RTB phenomenon, we need to predict the future position of the UCAV. Assume $\Delta T=$ $t_{k}-t_{k-1}=c \Delta t, \Delta t$ is one of the intervals between $t_{k}$ and $t_{k-1}$, and $c$ means the number of the intervals. We regard the whole process as a combination of uniform motion and accelerated motion. If the information at time $t_{k-1}$ is given, then the position in $X$ direction at time $t_{k}$ obeys Gauss distribution.

During $k$ th interval in time $\left[t_{k-1}, t_{k}\right]$, the position, velocity, and acceleration of UCAV $u_{i}$ in $X$ direction can be described as $x_{k}, v_{k}^{x}$, and $a_{i}^{x}$, and they have the following relationships:

$$
\begin{aligned}
& v_{k}^{x}=v_{0}^{x}+\sum_{i=0}^{k-1} a_{i}^{x} \Delta t, \\
& x_{k}=x_{0}+k v_{0}^{x} \Delta t+\frac{1}{2} \sum_{i=0}^{k-1}(2 k-2 i-1) a_{i}^{x} \Delta t^{2} .
\end{aligned}
$$

According to the definition of Gauss distribution, if the position of $u_{i}$ at $t_{k-1}$ in $X$ direction is given, we can set the Gauss variables of position at $t_{k}$ as: $\mu_{x}=E\left(x_{k}\right)$, and $\sigma_{x}^{2}=$ $D\left(x_{k}\right)$. And, after $\Delta T=t_{k}-t_{k-1}=m \Delta t$, we can get the probability

$$
\begin{aligned}
& p\left\{\left(x_{k}, y_{k}\right) \mid\left(x_{k-1}, y_{k-1}\right)\right\} \\
& \quad=\frac{1}{2 \pi \sigma_{x} \sigma_{y}} \exp \left\{\frac{-\left(x_{k}-\mu_{k}\right)^{2}}{2 \sigma_{x}^{2}}+\frac{-\left(x_{k}-\mu_{k}\right)^{2}}{2 \sigma_{x}^{2}}\right\} .
\end{aligned}
$$

Then, we can get the position at $t_{k}=t_{k-1}+m \Delta t$ as

$$
\begin{aligned}
& x_{k}=\text { predict_pos_} x\left(x_{k-1}, v_{k-1}^{x}, a_{k-1}^{x}, \Delta T\right), \\
& y_{k}=\text { predict_pos_} y\left(y_{k-1}, v_{k-1}^{y}, a_{k-1}^{y}, \Delta T\right) .
\end{aligned}
$$

\subsubsection{Rout Temporary Blindness Functional Avoidance (RTBFA) Model}

(a) Analyze the Mission: Positive and Negative Missions. In order to simplify the combat conditions, the missions have been divided into two different types, namely, positive mission $M_{P}$ and negative mission $M_{N}$.

Definition 1. One defines the positive mission as a preplanned mission which may be executed based on the possessed knowledge of the enemy. And the positive mission may have a hiding policy to reveal the information to the enemy as less as possible. In all, when executing a positive mission, the formation may have obtained the priority in combat.

According to Definition 1, one can see that when executing a positive mission, $\alpha=0$ or $\alpha<0$. The enemy may consider random attack policy or low degree node preferential policy. Neighbor node with low degree may become more vulnerable.

Definition 2. Negative mission is an unintentional or partial knowledge possessed mission; it happens when outer threats come out without prediction or inner malfunctions appear in some probability. During negative mission process, the enemy has occupied the combat situation and it has possessed more information of our formation. 
Analyzed by Definition 2, when executing a negative mission, $\alpha \rightarrow \infty$ or $\alpha>0$. The enemy may consider deliberate attack policy or high degree preferential policy. Neighbor node with high degree may become more vulnerable.

(b) Measurement of Possessed Information. The detective information is regarded as the possessed information about the enemies, and the detective problem is related to the hypothesis testing problem:

$$
\begin{aligned}
& H_{0} \text { : there is no object within the search range. } \\
& H_{1} \text { : there exist (an) object(s) within the search range. }
\end{aligned}
$$

Suppose that the probabilities are $P_{0}=P\left(H_{0}\right)$ and $P_{1}=$ $P\left(H_{1}\right)$ and the related variables of detective problem are as follows.

(i) Probability of False Alarm (PFA). It is the probability of a false decision which regards an event that did not happen as an event that happened. $P_{F}=\operatorname{Pr}\left(u=1 \mid H_{0}\right)$, and the PFA of airborne pulsed Doppler radar is [20]

$$
P_{F}=\int_{V_{T}}^{\infty} \frac{R}{\psi_{0}} \exp \left(-\frac{R^{2}}{2 \psi_{0}}\right) d R=\exp \left(-\frac{V_{T}^{2}}{2 \psi_{0}}\right),
$$

where $R$ is the amplitude of noise envelope from the detector, $\psi_{0}$ is the mean square value of noise voltage, and $V_{T}$ is threshold voltage.

(ii) Probability of the Right Decision. It is the probability of a right recognition and decision which can be described as

$$
P_{L}=\operatorname{Pr}\left(u=0 \mid H_{0}\right)=1-P_{F}=1-\exp \left(-\frac{V_{T}^{2}}{2 \psi_{0}}\right) .
$$

(iii) Probability of Detection. When an event happened and is correctly decided by the detective facility, the probability can be described as $P_{D}=\operatorname{Pr}\left(u=1 \mid H_{1}\right)$. And the PD of airborne pulsed Doppler radar is

$$
P_{D}=\int_{V_{T}}^{\infty} \frac{R}{\psi_{0}} \exp \left(-\frac{R^{2}+A^{2}}{2 \psi_{0}}\right) I_{0}\left(\frac{R A}{\psi_{0}}\right) d R
$$

(iv) Probability of Miss. It is the opposite meaning of PFA, that is, when an event did happen while the detective facility decides that it as an event that did not happen. $P_{M}=\operatorname{Pr}(u=$ $\left.0 \mid H_{1}\right)$, and the PM of airborne pulsed Doppler radar is

$$
\begin{aligned}
P_{M} & =1-P_{D} \\
& =1-\int_{V_{T}}^{\infty} \frac{R}{\psi_{0}} \exp \left(-\frac{R^{2}+A^{2}}{2 \psi_{0}}\right) I_{0}\left(\frac{R A}{\psi_{0}}\right) d R
\end{aligned}
$$

Under condition $H_{0}$, the information Shannon can be regarded as

$$
I_{0}=-\sum_{i=1}^{n} p_{i} \log _{2}\left(p_{i}\right)=-P_{F} \log _{2}\left(P_{F}\right)-P_{L} \log _{2}\left(P_{L}\right) .
$$

And, under condition $H_{1}$, the information Shannon can be regarded as

$$
I_{1}=-\sum_{i=1}^{n} p_{i} \log _{2}\left(p_{i}\right)=-P_{F} \log _{2}\left(P_{M}\right)-P_{D} \log _{2}\left(P_{D}\right) .
$$

The information Shannon [21] under detection condition is set as the sum of $I_{0}$ and $I_{1}$ :

$$
\begin{aligned}
I_{M}= & P\left(H_{0}\right) \cdot I_{0}+P\left(H_{1}\right) \cdot I_{1} \\
= & P_{0} \cdot\left[-P_{F} \log _{2}\left(P_{F}\right)-P_{L} \log _{2}\left(P_{L}\right)\right]+P_{1} \\
& \cdot\left[-P_{M} \log _{2}\left(P_{M}\right)-P_{D} \log _{2}\left(P_{D}\right)\right] .
\end{aligned}
$$

$I_{M}$ reflects the detective information. The bigger $I_{M}$ gets, the more the information is needed. Set an expected value $I_{E}$, and if $I_{M}>I_{E}$, the information is enough and the mission can be regarded as positive mission. If $I_{M}<I_{E}$, the mission can be regarded as a negative mission:

$$
\alpha=I_{E}-I_{M}
$$

\subsubsection{Avoidance Function}

$$
L_{i}= \begin{cases}1, & \text { others } \\ 0, & \text { if }\left(r_{i}-r_{0}\right) \|\left(W_{\alpha}\left(k_{i}\right)-W_{0}\right)<0\end{cases}
$$

$L_{i}$ is a decision function that measures the effectiveness of the current chosen nodes.

3.3. RTBA Utility Indexes. In order to get a more reliable communicating partner, we set the following indexes.

(i) Continuously connecting feature: $\Delta T=\max _{j \in I_{i}} \Delta T_{j}, I_{i}$ being the set of the neighbors of $u_{i}$.

According to the predicted position of UCAV $u_{0}$ and $u_{k}$, the relative position between the sender and receiver can be described as

$$
\Delta d=\sqrt{\left(x_{k}-x_{0}\right)^{2}+\left(y_{k}-y_{0}\right)^{2}} .
$$

After $\Delta T$, the positions of the sender and receiver are $\left(x_{0}^{\prime}, y_{0}^{\prime}\right)$ and $\left(x_{k}^{\prime}, y_{k}^{\prime}\right)$.

$$
\Delta d^{\prime}=\sqrt{\left(x_{k}^{\prime}-x_{0}^{\prime}\right)^{2}+\left(y_{k}^{\prime}-y_{0}^{\prime}\right)^{2}},
$$

where $\Delta d^{\prime}$ describes the connecting relationship between UCAV $u_{0}$ and $u_{k}$ after $\Delta T$ and $\Delta T$ can be regarded as continuously connecting feature.

(ii) Nearer connecting distance: $D=\min _{j \in s_{i}^{n}} D_{j}, D_{j}=$ $\sqrt{\left(x_{j}-x_{d}\right)^{2}+\left(y_{j}-y_{d}\right)^{2}}$.

The complex index can be described as

$$
\begin{aligned}
Q & =\min _{j \in s_{i}^{n}} Q_{j} \\
& = \begin{cases}\left(D_{j}+\frac{\tau}{\Delta T_{j}}\right) \cdot L_{i} \cdot W_{\alpha}\left(k_{j}\right), & \left(D_{j}<D_{i}\right) \wedge(\Delta T \geq 1) \\
\text { MAX_VALUE, } & \text { otherwise, }\end{cases}
\end{aligned}
$$

where $\tau$ is the reliability weight of neighbor nodes. 
The connecting result of each node can be decided by the value of $Q$, and we can finally get a neighbor matrix $m$ which decides the structure of the formation. According to the features of complex networks, if more than two nodes need to be replaced during the same mission period, then the new nodes choosing order may be very important to the net structure. Different nodes choosing order may lead to different neighbor matrix. $m_{i} \in M$ is the neighbor matrix set, and we need to get a proper matrix which can bring a more stable and effective result.

\section{Dynamic Topology Optimizing Method Based on Synchrony Features}

4.1. The Synchronization of UCAV Formation. Synchronization phenomenon exists widely in nature, such as the synchronization of fireflies glowing and the synchronization of large groups of neurons in human brains and the clapping frequency synchronization [22]. The synchronization phenomenon is also an important concept in UCAV formation and it reflects the recognizing abilities of each node and we give the definition of synchronization in formation.

Definition 3. Synchronization in UCAV formation can be regarded as the agreement on the combat situation by different UCAV nodes. And it can be described by the conformance degree towards the same combat situation.

As one mentioned in Section 3.1, each UCAV node can be regarded as a dynamic system, and there exists coupling effect between two connected nodes. Then the dynamic equation of each node can be given as below:

$$
\dot{x}_{i}=F\left(x_{i}\right)-\sigma \sum_{j}^{N} l_{i j} \mathbf{H} x_{j}, \quad i=1,2, \ldots, N,
$$

where $x_{i} \in \mathbf{R}^{d}$ is a $d$ dimensional state vector and $F: \mathbf{R}^{d} \rightarrow$ $\mathbf{R}^{d}$ is the dynamic equation which obeys Lipchitz condition [23].

$\sigma$ is coupling efficient, $\mathbf{H}$ describes the different coupling styles among different vibrators, $l_{i j}$ is the element with Laplace features, and $x_{i}(t)(i=1,2, \ldots, N)$ is the state of each node.

If any solution to (20) satisfies the following condition, then we can say that the system is a completely synchronized system:

$$
\lim _{t \rightarrow \infty}\left\|x_{i}(t)-x_{j}(t)\right\|=0, \quad i, j=1,2, \ldots, N .
$$

If there exists a real number $\varepsilon$ which is greater than zero, it constructs the following relationship:

$$
\lim _{t \rightarrow \infty}\left\|x_{i}(0)-x_{j}(0)\right\|<\varepsilon .
$$

If any solution to the equation above satisfies the following condition, then we can say that the system is a local synchronized system:

$$
\lim _{t \rightarrow \infty}\left\|x_{i}(t)-x_{j}(t)\right\|=0, \quad i, j=1,2, \ldots, N .
$$

The UCAV formation can be regarded as a local synchronization system in this research.
4.2. Indexes of Synchronization in UCAV Formation. The synchronization of UCAV formation is decided by the following three factors [24]:

(i) The dynamic features of each node.

(ii) The net structure, namely, matrix $m$.

(iii) The coupling effect between different dynamic systems.

According to [24], $K=\lambda_{\max } / \lambda_{2}$ can be used to describe the synchronization features. $\lambda_{\max }$ describes the robustness towards the communication delay and $\lambda_{2}$ means the second largest eigenvalue which can also be regarded as algebraic connectivity. The synchronization feature of the system is often improved by decreasing the value of $K . \lambda_{i}$ in ascending sort order is the eigenvalue of matrix $m$. The evaluating function is set as

$$
E(G)=\left(\frac{\lambda_{2}}{\lambda_{\max }}\right)_{G} \sum_{R}\left(w_{r} \sum_{k} e\left(k_{i}\right)\right),
$$

where $\left(\lambda_{2} / \lambda_{\max }\right)_{G}$ is used to evaluate the connectivity and delay robustness of UCAV formation, $R$ is the number of kinds of functional nodes, $w_{r}$ means the weight in relation to different kinds of functional nodes, $\sum_{R} w_{r}=1$, and $\sum_{k} e\left(k_{i}\right)$ is the sum of all the nodes' efficiencies. From [25], we can get the node's efficiency model:

$$
e\left(k_{i}\right)=\frac{e^{\left(\nu-\beta k_{i}\right)}}{1+e^{\left(\nu-\beta k_{i}\right)}},
$$

where $k_{i}$ is the degree of node $i$ and $\nu$ and $\beta$ are the modifying variables according to the situation. In order to get an optimal value, we need to get $\max (E(G))$. Then the optimal function can be set as

$$
\begin{array}{ll}
\max & (E(G))=\left(\frac{\lambda_{2}}{\lambda_{\max }}\right)_{G} \sum_{R}\left(w_{r} \sum_{k} e_{r}\left(k_{i}\right)\right) \\
\text { s.t. } & \sum_{R} w_{r}=1 .
\end{array}
$$

4.3. Dynamic Topology Constructing Method Based on HPSO Method. According to the aforementioned contents, the optimal structure needs to be found in order to keep the stability and synchrony of the UCAV system. The new nodes choosing order has brought more than one kind of net constructing structure. Thus the problem has been simplified as finding a proper sequence.

4.3.1. Calculating Process. According to the problem simplification above, we have changed the complex model into a typical TSP model; thus we use hybrid particle swarm optimization method to solve this problem. The detailed steps can be acquired in Figure 5.

4.3.2. Encoding Method. The length of the code equals the number of nodes which need to find new partners. The codes can be described by natural numbers which 


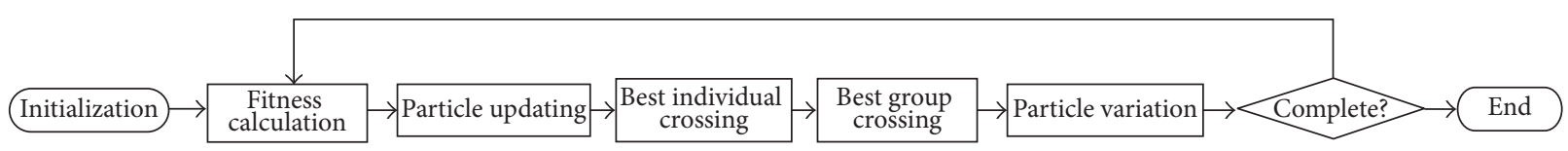

FIgURE 5: Calculating steps.

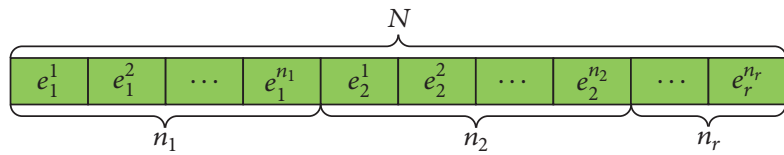

FIGURE 6: Encoding method.

should get repeated. If the new nodes choosing order is $\{1,2,5,6,9,7,8,3,4, \ldots, n\}$, then the code can be set as $\{1,2,5,6,9,7,8,3,4, \ldots, n\}$.

In Figure 6, $e_{r}^{n_{r}}$ is the sequence number which means the sequence of the node $n_{r}$ in $r$ kind of functional node. Different kinds of functional nodes may match different kinds of weights which are very important to the final result.

4.3.3. Code Crossing Rules. In order to accelerate the process of convergence, the crossing rules are brought into the algorithm. And the detailed rules are described in Figure 7.

From Figure 7, the crossing rules are as follows:

(i) Selecting two crossing points in the old code set randomly.

(ii) Exchanging the codes between the crossing points.

(iii) Executing the self-testing process to avoid the repeated codes.

(iv) Rebuilding the codes.

\section{Simulation}

5.1. Hardware Condition. Hardware condition is as follows: CPU: P4 2.8 GHz, RAM: 1.5 G, and Matlab 2010, NS2.

\subsection{Software Settings}

Variables

Values

Simulation range

$100 \mathrm{~km}^{2}$

UDP data

512 Bytes

Transportation fading model

Friis

UCAV communicating distance $\quad 1 \mathrm{~km}$

Communication treaty UDP

Simulation time

$200 \mathrm{~s}$

Simulation times

1000

5.3. Evaluation Indexes. In order to verify the effectiveness of RTBA model, we use Packet Delivery Ratio (PDR) and Endto-End Delay (EED) to complete the evaluating process.

\subsubsection{Packet Delivery Ratio (PDR): Ratio of Information Received by All the Possible Receiving Nodes to Information Sent by an Exact Sending Node}

$$
\mathrm{PDR}=\frac{\sum_{n} D_{r}}{D_{s}} .
$$

$D_{r}$ is the information received by a chosen node, $\sum_{n} D_{r}$ describes the information received by $n$ possible chosen nodes. $D_{s}$ describes all the sent information.

\subsubsection{Average End-to-End Delay (AEED): The Average Time of Information Transportation from Sender to Receivers}

$$
T_{D}=\frac{\sum_{i=1}^{n}\left(T_{i}^{r}-T_{i}^{s}\right)}{n},
$$

where $T_{i}^{r}$ means the time when the $i$ data package is received, $T_{i}^{s}$ means the time when the $i$ data package is sent, and $n$ is the number of data packages.

\subsection{Simulation and Analysis}

5.4.1. RTBA Model Analysis. Three models are put forward to compare the PDR values. Here we used RTBA, RTBPA, and normal model to make a comparison while the normal model is the traditional communication model without any restoring strategies. We first assume that the mission has kept a stable status, and the UCAV nodes fly at an increasing velocity.

The initial number of UCAV nodes is 50 in Figure 8(a), and we can find that during a stable status, the RTBA model may have a better PDR value. With the increasing of the velocity of nodes, the robustness of UCAV formation may be influenced. The continuously increasing velocity may enlarge the error in RTB problem, so the PDR may decrease, but RTBA model still has a better performance than others. And, in this situation, RTBPA model has almost the same performance as RTBA. Figure 8 (b) has the same simulating environment but an increasing UCAV number. We set 100 UCAV nodes in the right side. We can easily find that the increasing number of UCAVs may have an obvious superiority. At a relatively low velocity, the normal model may even have a better value than the others, but, with the increasing of the velocity, RTBA model will show its superiority.

In order to describe the performance of models in an unstable status, a sudden threat is designed at time $30 \mathrm{~s}$, and then negative mission $M_{N}$ appears. The velocity of UCAV formation is given as $100 \mathrm{~m} / \mathrm{s}$ and the PDR values are shown in Figure 9. 


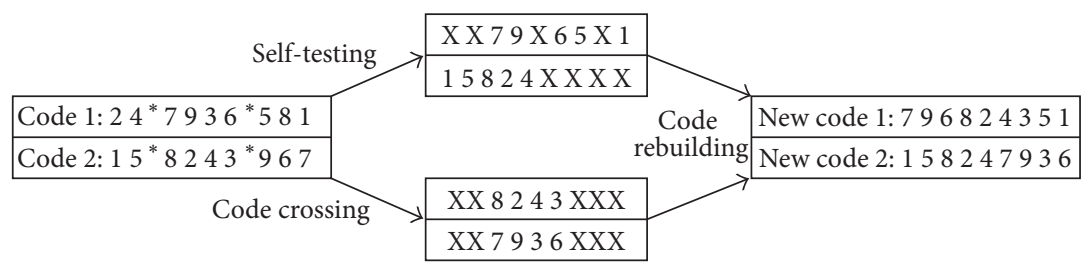

FIgURE 7: Crossing method.

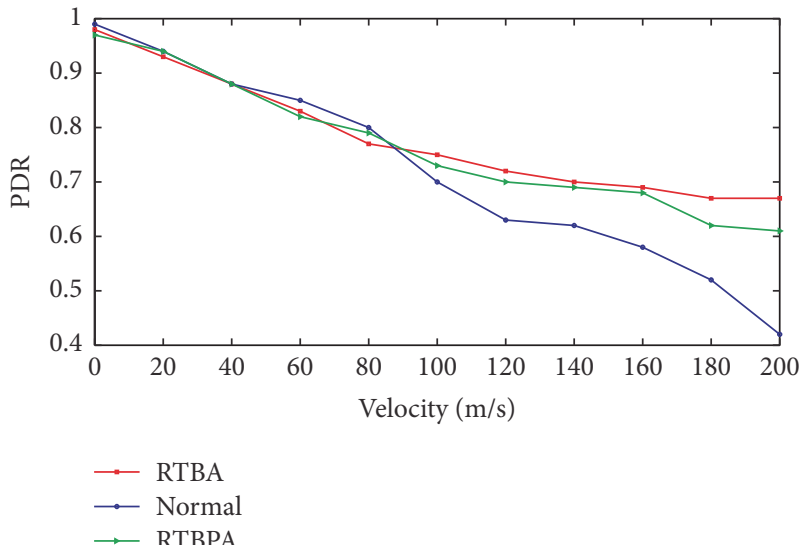

(a)

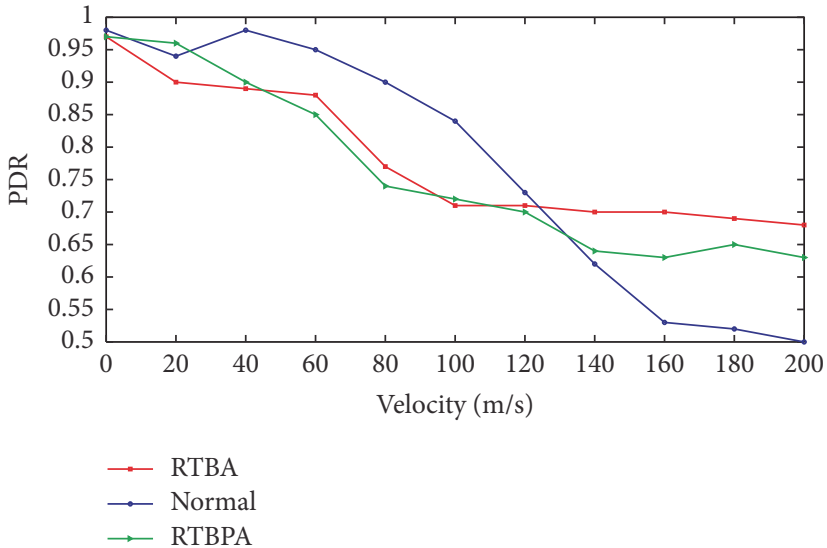

(b)

FIGURE 8: PDR comparison among different models.

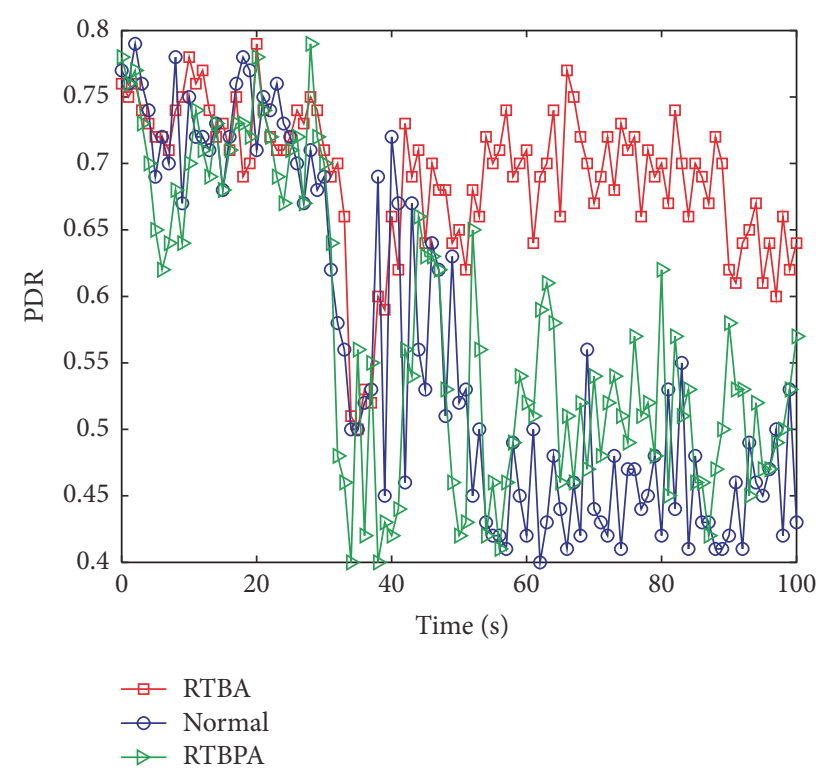

FIGURE 9: PDR comparison when sudden threat comes at time $30 \mathrm{~s}$.

Figure 9 reflects the PDR comparison when executing $M_{N}$, and the unexpected threat has influenced the robustness of the formation. After $30 \mathrm{~s}$, the threat has enlarged the potential risk of parts of the nodes, then PDR in all the models have descended. At about $45 \mathrm{~s}$, PDR in RTBA model has bounced back and kept a stable value, while PDR in normal and RTBPA model has not bounced back. The threat has great influences on the functional feature of the nodes; RTB model may have a better self-testing and restoring ability which can be important to keep the robustness of UCAV formation.

AEED can also be used to evaluate the efficiency of a cooperative system. From Figure 10, RTBA model has a relatively low AEED value with the increasing of the velocity. Figure 10(a) has 50 nodes and Figure 10(b) has 100 nodes and Figure 10(a) has an obvious low average value compared to that in Figure 10(b). More nodes may offer more connecting choices, and more choices may shorten the node choosing time, so the average value of AEED is lower. The increasing velocity may enlarge the physical error and the formation in RTBA and RTBPA model has to test the connectivity of the system for many times, and then the value of AEED increases.

Figure 11 shows the average time delay probability in different models after 1000 times' simulations when the formation encounters a sudden threat (negative mission). For simplification, we make the time delay remain at one decimal. In RTBA model, the time delay focus on 0.2 to 0.4 seconds. In RTBPA model, the time delay focus on 0.4 to 0.8 seconds. In normal model, though the time delay shows its random features, it has a greater average value. And we can find the following: $\sum p_{\mathrm{RTBA}}=1, \sum p_{\mathrm{RTBPA}}<1$, and $\sum p_{\text {Normal }}<1$. Among 1000 simulations, there exists communicating broken situation in RTBPA and normal models. If a communicating broken problem happens, value of AEED may trend to be infinite and the result may be totally different.

5.4.2. Optimal Synchronized Structure Seeking. The superiority has been analyzed in Section 5.4.1; then the optimal structure should be acquired in Section 5.4.2. 


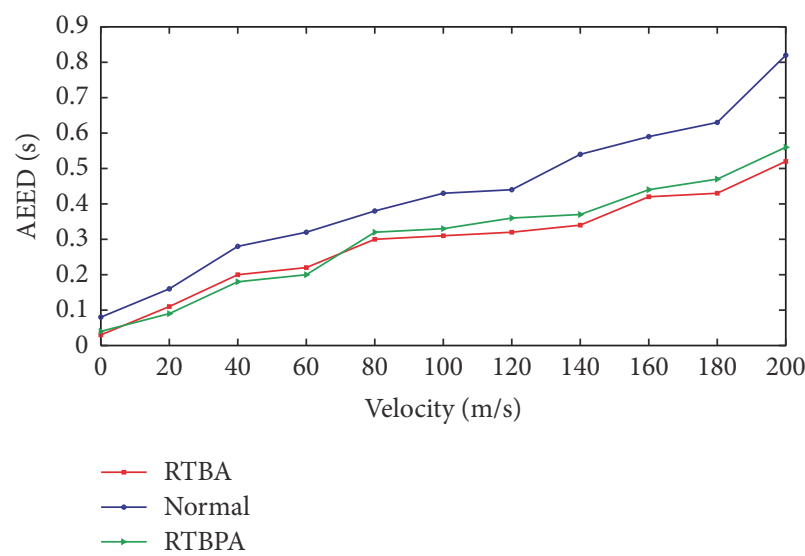

(a)

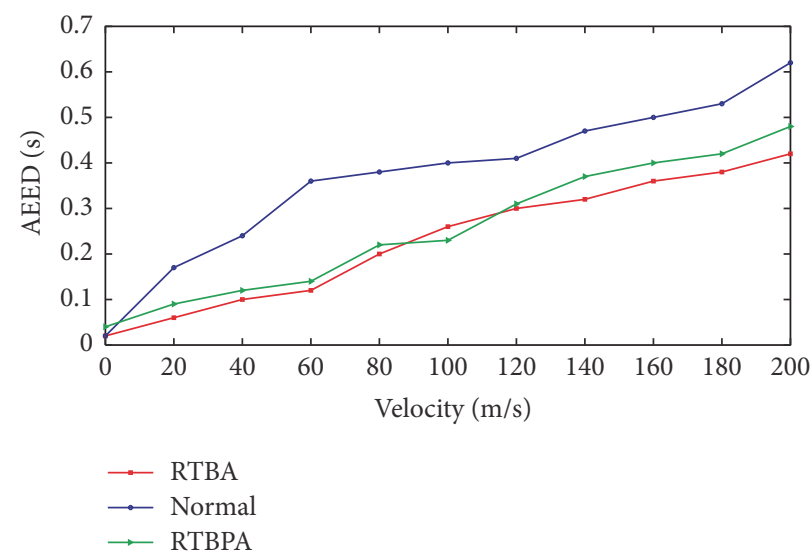

(b)

FIGURE 10: AEED comparison among different models.

TABLE 1: Initialization of UCAV structure.

UCAV number

Kinds of functional nodes

Mission stage

$\lambda_{\max } / \lambda_{2}=21.221$

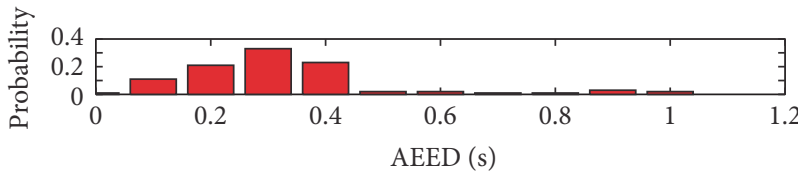

RTBA

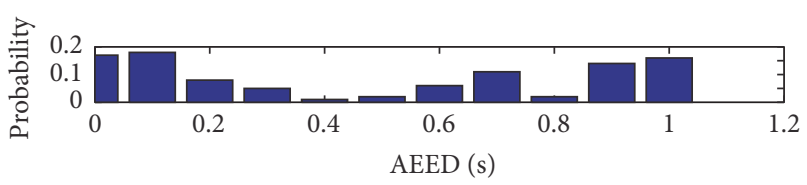

Normal

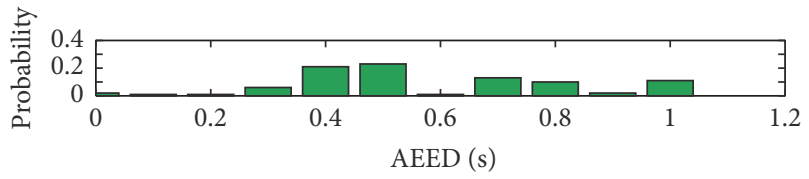

- RTBPA

FIGURE 11: AEED probabilities of three models under sudden threats.

The initial structure can be found in Figure 12 and the values of features can be seen in Table 1 .

We assume that, after the appearance of a sudden threat, nodes 2, 6, and 9 may get a higher probability of being attacked, and then a better node choosing order is needed so as to get a better constructing structure.

From Figure 13, HPSO has an obvious better result than PSO, the best node choosing order is as follows: bestSequence $=(4 \rightarrow 19 \rightarrow 18 \rightarrow 5 \rightarrow 2 \rightarrow 1 \rightarrow 10 \rightarrow$ $8 \rightarrow 11 \rightarrow 17)$, and the optimized structure can be found in Figure 14.
21 (20 communication nodes in relative UCAV platform) table stages, 1 mission changing stage (sudden threat comes at $30 \mathrm{~s}$ )

$1 / \lambda_{2}=2.447 \quad e=38$ (edges)

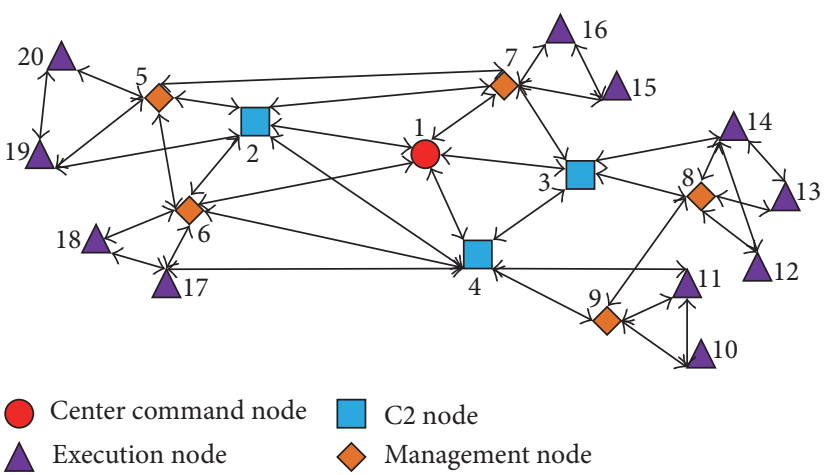

FIGURE 12: Description of initial net structure.

From Figure 14 we can see that the edges among different nodes have increased, and the edges around nodes 2, 6, and 9 have decreased. The whole formation has a flatter structure which contains more cross-grade connections. And the relative values of relative variables are as follows: $1 / \lambda_{2}=$ $1.718, \lambda_{\max } / \lambda_{2}=16.702, e=44$, and $E=0.724$. The value of $\lambda_{\text {max }} / \lambda_{2}$ has decreased due to the interception of the negative mission. The result can also describe that a flat structure may be proper to improve the synchrony of UCAV formation.

\section{Conclusions}

Dynamic UCAV formation net constructing problem under RTB situation is solved in this paper. Robustness and synchronization are set as two important indexes to seek for an optimal UCAV formation structure. In order to analyze the influences caused by the combat missions, the mission kinds and mission stages are classified, and a dynamic structure 


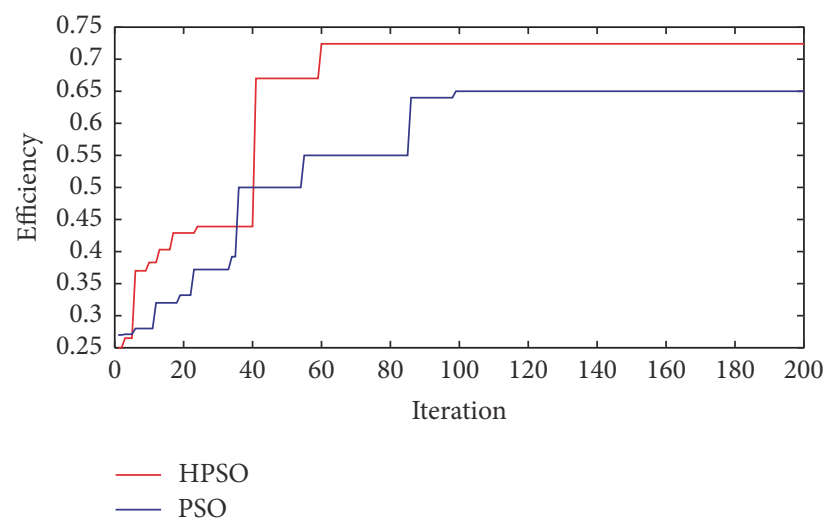

FIGURE 13: Comparison between PSO and HPSO algorithm.

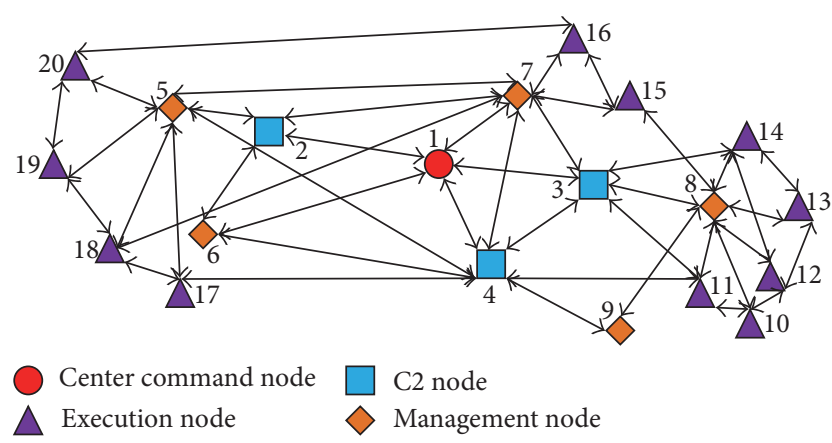

FIGURE 14: Optimized structure of UCAV formation.

changing mechanism is put forward. RTBA model is given to optimize the functional and physical nodes in order to keep the robustness of the formation. And HPSO method is used to find a proper structure to get an appropriately synchronized features. The dynamic structure constructing problem has been transferred to a node choosing sequence optimizing problem which can be regarded as a TSP problem. Finally, the efficiency of RTBA model has been given according to the comparison with other models and the optimal structure with better robustness and synchronization has been acquired using HPSO method.

From the perspective of net constructing consumption, the optimized formation structure is able to keep a synchronized feature with not many changes in edges. This will be very important to decrease the cost of keeping the connections among different UCAVs.

From the perspective of robustness, the high risky nodes have relatively low probability of being connected in the optimized structure which can be useful to keep the robustness of the formation.

From control and command perspective, edges in formation have increased obviously, and the cross-grade connections have taken a great ratio in the optimized structure. A flatter formation is useful to improve the comprehensive efficiency of UCAV formation.

Combat situation is very important because it can influence the state of UCAV and even the structure of UCAV formation. In this content, combat situation is simple and the scenario is regarded as an absolutely ideal space. A complex scenario may be more credible in real combat situation. Thus we need to pay more attention to this area in order to put the theory into practice.

\section{Competing Interests}

The authors declare that they have no competing interests.

\section{References}

[1] C. Pan, W. Ye, J. Chen, and J. Guo, "Cooperative control for UCAVs path planning," in Proceedings of the 8th World Congress on Intelligent Control and Automation (WCICA '10), pp. 52595263, Jinan, China, July 2010.

[2] J. Chen and X. Gao, "Modeling and analysis of command and control system in cooperative air combat," Engineering and Applications, vol. 45, no. 10, pp. 195-198, 2009.

[3] A. H. Levis, Time Sensitive Control of Air Combat Operations, Cebter of Excellence in Command, Control, Communication and Intelligence, George MASON University, 1998.

[4] F. D. J. Bowden, Petri Nets and Their Application to Command and Control Systems, Information Technology Division, Electronics and Surveillance Research Laboratory, Salisbury, Australia, 1996.

[5] S. Erkan, M. Kandemir, and G. Giger, "Advanced task assignment for unmanned combat aerial vehicles targeting cost efficiency and survivability," in Proceedings of the 46th AIAA Aerospace Sciences Meeting and Exhibit, Reno, Nev, USA, January 2008.

[6] C. Schumacher, P. Chandler, P. Pachter, and L. Pachter, "Constrained optimization for UAV task assignment," in Proceedings of the AIAA Guidance, Navigation, and Control Conference and Exhibit, Guidance, Navigation, and Control and Co-located Conferences, Providence, RI, USA, August 2004.

[7] D. Zhang, G. Xie, J. Yu, and L. Wang, "Adaptive task assignment for multiple mobile robots via swarm intelligence approach," Robotics and Autonomous Systems, vol. 55, no. 7, pp. 572-588, 2007.

[8] T.-Q. Ni, J.-D. Wang, and Y.-A. Liu, "Application of particle swarm algorithm in route planning of UAV," Systems Engineering and Electronics, vol. 33, no. 4, pp. 806-810, 2011.

[9] R. J. Szczerba, P. Galkowski, I. S. Glicktein, and N. Ternullo, "Robust algorithm for real-time route planning," IEEE Transactions on Aerospace and Electronic Systems, vol. 36, no. 3, pp. 869-878, 2000.

[10] S.-Q. Tang, C.-Q. Huang, J. Hu, and W.-C. Wu, “Threat equivalent and improved PSO algorithm based real-time method of UCAV route planning," Systems Engineering and Electronics, vol. 32, no. 8, pp. 1706-1710, 2010.

[11] J.-Y. Du, F.-M. Zhang, H.-B. Mao, H.-W. Liu, and J. Yang, "Game theory based multi-UAV cooperative searching model and fast solution approach," Journal of Shanghai Jiaotong University, vol. 47, no. 4, pp. 667-678, 2013.

[12] B. Di, R. Zhou, and Q.-X. Ding, "Distributed coordinated heterogeneous task allocation for unmanned aerial vehicles," Control and Decision, vol. 28, no. 2, pp. 274-278, 2013.

[13] X. K. Bao, Modeling and Analyzing of Networked Operation Synchronization, National University of Defense Technology, Changsha, China, 2009. 
[14] M. M. Zavlanos, L. Spesivtsev, and G. J. Pappas, "A distributed auction algorithm for the assignment problem," in Proceedings of the 47th IEEE Conference on Decision and Control (CDC '08), pp. 1212-1217, Cancun, Mexico, December 2008.

[15] D. Medina, F. Hoffmann, F. Rossetto, and C.-H. Rokitansky, "Routing in the airborne internet," in Proceedings of the Integrated Communications, Navigation, and Surveillance Conference (ICNS '10), pp. 11-13, Herndon, Va, USA, May 2010.

[16] X. Huang, Scientific Research on Complex Methodology, Qsinghua University, Beijing, China, 2014.

[17] S. Boccaletti, V. Latora, Y. Moreno, M. Chavez, and D.-U. Hwang, "Complex networks: structure and dynamics," Physics Reports, vol. 424, no. 4-5, pp. 175-308, 2006.

[18] R. John, Dynamic Complexity in System of Systems, Engineering \& Technology. Advanced Sytems. Boeing Company, 2007.

[19] L. K. Gallos, R. Cohen, P. Argyrakis, A. Bunde, and S. Havlin, "Stability and topology of scale-free networks under attack and defense strategies," Physical Review Letters, vol. 94, no. 18, Article ID 188701, 4 pages, 2005.

[20] D. Luo, Z. Yang, H. Duan, Z. Wu, and C. Shen, "Heuristic particle swarm optimization algorithm for air combat decisionmaking on CMTA," Transactions of Nanjing University of Aeronautics \& Astronautics, vol. 23, no. 1, pp. 20-26, 2006.

[21] D. Stein, J. Witkoskie, S. Theophanis, and W. Kuklinski, "Random set tracking and entropy based control applied to distributed sensor networks," in Proceedings of the Proceedings of SPIE, vol. 6567, pp. 656709-656722, Beijing, China, November 2007.

[22] S. Li, Research on the Mechanism of Cascading Dynamics and Behaviors of Complex Networks, Beijing University of POSIS and Telecommunications, 2012.

[23] D. Shi, G. Chen, W. W. K. Thong, and X. Yan, "Searching for optimal network topology with best possible synchronizability," IEEE Circuits and Systems Magazine, vol. 13, no. 1, pp. 66-75, 2013.

[24] M. Jalili, "Enhancing synchronizability of diffusively coupled dynamical networks: a survey," IEEE Transactions on Neural Networks and Learning Systems, vol. 24, no. 7, pp. 1009-1022, 2013.

[25] J. Yan, X.-M. Li, L.-J. Liu, and B. Liu, "Combat network synchronization of air fleet formation," Systems Engineering and Electronics, vol. 36, no. 8, pp. 1566-1572, 2014. 


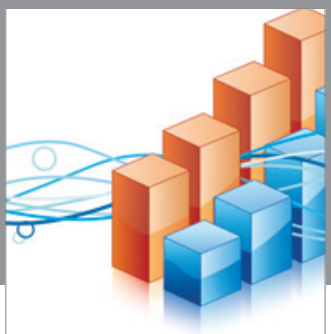

Advances in

Operations Research

vatem alat4

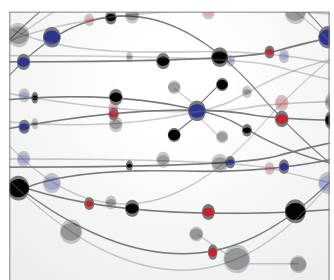

\section{The Scientific} World Journal
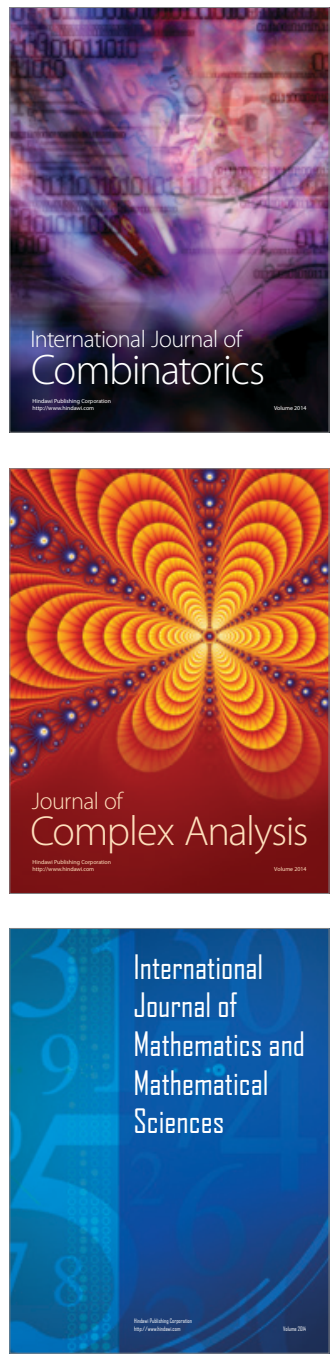
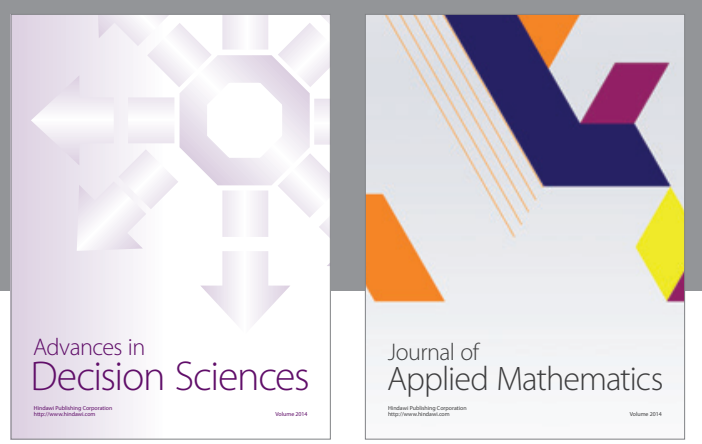

Algebra

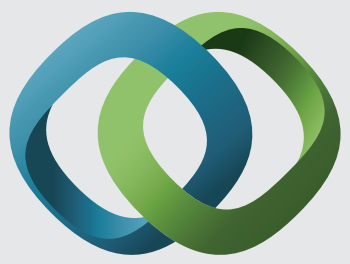

\section{Hindawi}

Submit your manuscripts at

http://www.hindawi.com
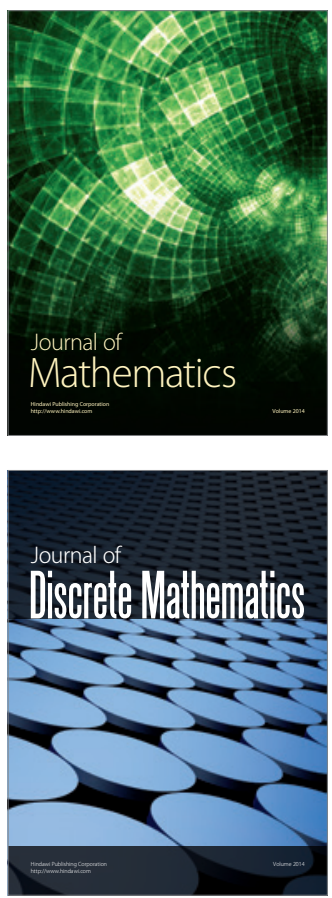

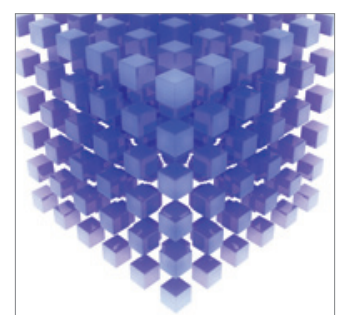

Mathematical Problems in Engineering
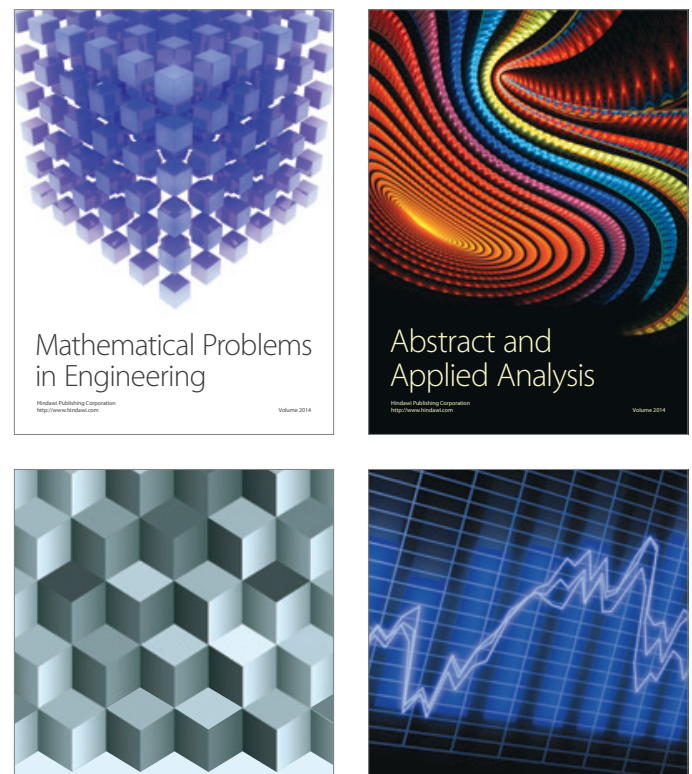

Journal of

Function Spaces

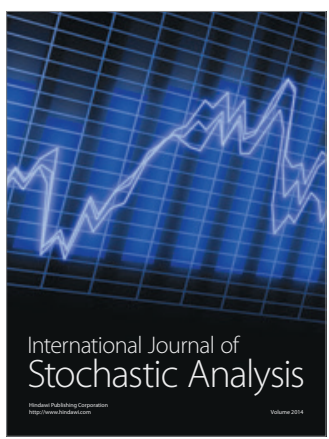

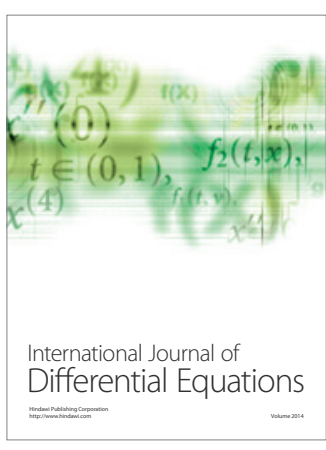
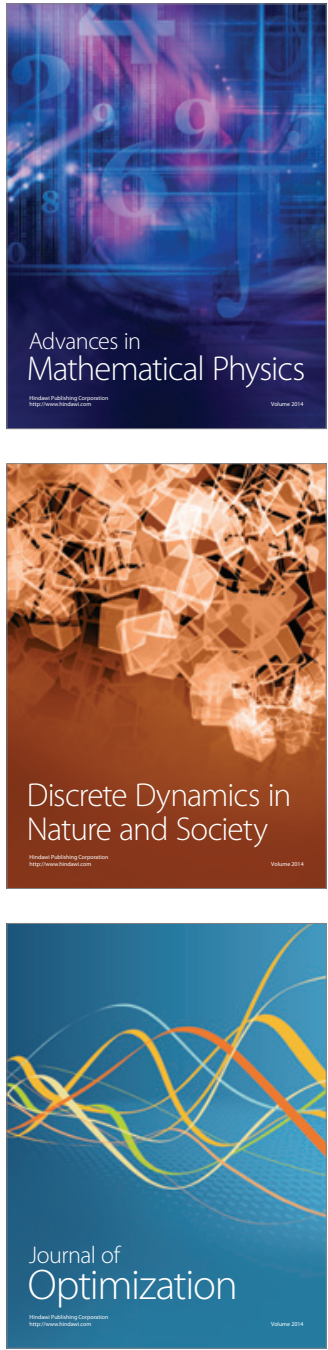